\title{
球面之间的双 $\mathbb{Z}_{3}$ 等变映射
}

\author{
唐梓洲 \\ (中国科学院数学研究所, 北京 100080 )
}

\section{关锚词 $\mathrm{Z}_{p}$ 等变映射、模 $d$ 的 Dold 定理、 $\tilde{J}$ 群}

设 $S^{2 n+1}$ 为 $(n+1)$ 维复欧氏空间 $\mathrm{C}^{n+1}$ 中的标准球面. 设 $T: S^{2 n+1} \rightarrow S^{2 n+1}$ 是一个由 下式定义的变换: $T\left(z_{0}, z_{1}, \cdots, z_{n}\right)=\left(e^{\frac{2 \pi i}{\rho}} z_{0}, e^{\frac{2 \pi i}{\rho}} z_{1}, \cdots, e^{\frac{2 \pi i}{\rho}} z_{n}\right)$, 这里 $z_{0}, z_{1}, \cdots, z_{n}$ 为复 数, 满足 $\sum_{i=0}^{\infty}\left|z_{i}\right|^{2}-1$. $T$ 生成一个阶 $P$ 的循环群, 且在 $S^{2 n+1}$ 上自由作用, 其轨道空间即为 标准的 Lens 空间 $L^{*}(p)$.

定义 称映射 $f: S^{2 n+1} \rightarrow S^{2 n+1}$ 为 $Z_{p}$ 等变映射, 如果 $f(T x)-T f(x)$, 对任意 $x \in S^{2 n+1}$ 成立. 称映射 $f: S^{2 k+1} \times S^{2 n+1} \rightarrow S^{2 n+1}$ 为双 $Z$, 等变映射, 如果 $f$ 在每个分量上的限制都是 $\mathbf{Z}$, 等变映射.

引理 1 若存在 $f: S^{2 k+1} \times S^{2 n+1} \rightarrow S^{2 n+1}$ 为双 $Z_{p}$ 等变映射, 则必有 $k \leqslant n$.

证 将 $f$ 限制在第一个变量上, 得 $f_{1}: S^{2 k+1} \rightarrow S^{L s+1}$ 为 $Z_{p}$ 等变映射, 则由模 $p$ 的 BorsukUlam 定理(见文献 [1]), 必有 $k \leqslant n$.

引理 2 若 $f: S^{2 n+1} \rightarrow S^{2 n+1}$ 为 $Z_{p}$ 等变映射, 那么 $f$ 的映射度 $\operatorname{deg} f \neq 0 \bmod p$.

证由文献 [1] 中定理 1 .

下列引理是 Adams 证明的模 $d$ 的 Dold 定理.

引理 3 (见文献 [2] 中定理 1.1) 设 $\xi 、 \eta$ 为有限 $\mathrm{CW}$ 复形 $X$ 上的球面丛,如果存在保 纤维的映射 $f: E(\xi) \rightarrow E(\eta)$, 使 $f$ 在每一纤维上度为 $\pm d$, 则存在整数 $e \geqslant 0$, 使 $d^{*} \xi$ 与 $d^{e} \eta$ 为纤维同伦等价.

下面对 $p=3$ 的特殊情形来考虑. 设 $\pi: L^{k}(3) \rightarrow \mathrm{C} p^{k}$ 为典则投影, $\eta^{\prime}$ 为 $\mathrm{C} p^{k}$ 的 Hopf 复线丛, 记 $\eta-\pi^{*} \eta^{\prime}$ 为诱导丛, $\sigma=\eta-1_{\mathrm{c}} \in \widetilde{K}\left(L^{k}(3)\right)$, 易知 $(1+\sigma)^{3}-1$. 设 $r: \widetilde{K}\left(L^{k}(3)\right) \rightarrow \widetilde{K O}\left(L^{k}(3)\right)$ 和 $C: \widetilde{K O}\left(L^{k}(3)\right) \rightarrow \widetilde{K}\left(L^{k}(3)\right)$ 分别为实化映射与复化同态. 关于 $L^{k}(3)$ 的 $\widetilde{K O}$ 解, $\mathrm{Kambe}^{[3]}$ 指出:

引理 4 (1) 设 $k \neq 0 \bmod 4$, 则 $\widetilde{K O}\left(L^{k}(3)\right) \cong Z_{3}\left[\frac{k}{2}\right]$ 由 $\eta$ 生成. (2)设 $k \neq 0 \bmod 4$, 则 复化同态 $C: \widetilde{K O}\left(L^{k}(3)\right) \rightarrow \widetilde{K}\left(L^{k}(3)\right)$ 是单的, 且 $C(r \sigma)-\sigma^{2} / 1+\sigma$.

定理 1 设 $k \neq 0 \bmod 4$, 则 $\tilde{J}\left(L^{k}(3)\right) \cong \tilde{K O}\left(L^{k}(3)\right)$.

对 $x$ 为任一整数, 定义 $\nu_{p}(x)=s$, 如果 $p^{s}$ 整除 $x$, 但 $p^{s+1}$ 不能整除 $x$. 文献 [4] 中定 理 2 指出, 若存在 $S^{k-1} \times S^{n-1} \rightarrow S^{n-1}$ 为双 $Z_{c}$ 等变映射, 则

本文 1990 年 5 月 25 日收到. 


$$
v_{2}(n) \geqslant \Phi(k-1)-: \#\{0<x \leqslant k-1 \mid x \equiv 0,1,2,4 \bmod 8\} .
$$

若将双 $Z_{2}$ 等变映射改为 $Z_{3}$ 等变映射, 那么情形会怎么样呢? 我们的下列定理表明:

定理 2 设 $k \neq 0 \bmod 4$, 则若存在 $f: S^{2 k+1} \times S^{2 n+1} \rightarrow S^{2 n+1}$ 为双 $Z_{3}$ 等变映射, 那么必 有 $v_{3}(n+1) \geqslant\left[\frac{k}{2}\right]$.

我们先来证明定理 1 .

设 $\psi_{R}^{k}: \widetilde{K O}\left(L^{k}(3)\right) \rightarrow \widetilde{K O}\left(L^{k}(3)\right)$ 和 $\psi_{c}^{i}: \widetilde{K}\left(L^{k}(3)\right) \rightarrow \widetilde{K}\left(L^{k}(3)\right)$ 分别为实的和复的 Adams 运算, 它们都为环同态. 由于 $\psi_{c}^{i}(1+\sigma)=(1+\sigma)^{i}$, 于是利用 $(1+\sigma)^{3}=1$ 有

$$
c \cdot \psi_{k}^{2}(r \sigma)-\psi_{c}^{2} \cdot C(r \sigma)-\phi_{c}^{2}\left(\frac{\sigma^{2}}{1+\sigma}\right)=(1+\sigma)^{2}+(1+\sigma)-2-c(r \sigma) \text {, 由引理 }
$$

4(2) 可得

$$
\boldsymbol{\phi}_{R}^{2}(r \sigma)-r \sigma .
$$

由于

$$
\begin{aligned}
C\left(\psi_{R}^{33+2}(r \sigma)\right) & -\psi_{c}^{3 s+2}(C(r \sigma))-\frac{\left((1+\sigma)^{3 s+2}-1\right)^{2}}{(1+\sigma)^{3+2}} \\
& -\frac{\left((1+\sigma)^{2}-1\right)^{2}}{(1+\sigma)^{2}}-C\left(\psi_{R}^{2}(r \sigma)\right)-C(r \sigma),
\end{aligned}
$$

故同样可得 $\psi_{R}^{3 s+2}(r \sigma)-r \sigma$, 同样 $\psi_{R}^{3 s+1}(r \sigma)-\psi_{R}^{1}(r \sigma)=r \sigma$. 因此, 对一切 $i>0$ 都有

$$
\psi_{R}^{i}(r \sigma)-r \sigma=0 .
$$

设 $\tilde{J}^{\prime \prime}\left(L^{k}(3)\right)$ 为 $\operatorname{Adam} s^{i j}$ 定义的 $\tilde{J}^{\prime \prime}$ 群, 由以上结果, 可知 $\tilde{J}^{\prime \prime}\left(L^{k}(3)\right) \cong \widetilde{K O}\left(L^{k}(3)\right)$. 再由文献 [6]有 $\tilde{J}\left(L^{k}(3)\right) \cong \tilde{J}^{\prime \prime}\left(L^{k}(3)\right) \cong \tilde{N}^{\prime} O\left(L^{k}(3)\right)$.

下面我们来证明定理 2.

给定 $f: S^{2 k+1} \times S^{2 n+1} \rightarrow S^{2 n+1}$ 为双 $Z_{3}$ 等变媵射, 我们对

$$
(x, y) \in S^{2 k+1} / Z_{3} \times S^{2 n+1}=L^{k}(3) \times S^{2 n+1}
$$

定义 $F(x, y)-(x, f(x, y)) \in S^{2 k+1} \times S^{2 n+1} / Z_{3}$, 这里 $S^{2 k+1} \times S^{2 n+1} / Z_{3}$ 是由

$$
T(x, z)=(T x, T z)
$$

定义的变换 $T: S^{2 k+1} \times S^{2 n+1} \rightarrow S^{2 k+1} \times S^{2 n+1}$ 的轨道空间.

$L^{k}(3) \times S^{2 n+1}$ 即为 $L^{k}(3)$ 上 $(2 n+2)$ 维平凡丛的球面丛, 而 $S^{2 k+1} \times S^{2 n+1} / Z_{3}$ 即为 $L^{k}(3)$ 上二维向量丛 $r \sigma$ 的 $(n+1)$ 个 Whitney 和 $(n+1) r \sigma$ 的球面丛 $S((n+1) r \sigma)$. 由于 $f$ 为双 $Z_{3}$ 等变映射, 所以 $F$ 在每个纤维上是 $S^{2 n+1} \rightarrow S^{2 n+1}$ 的 $Z_{3}$ 等变映射, 设 $F$ 在每个 纤维上映射度为 $d$, 由引理 $2, d \not \equiv 0 \bmod 3$. 再根据引理 3 , 存在 $e \geqslant 0$ 使 $d \cdot c S(n+1) r \sigma$ 与 平凡球面众为纤维同伦等价, 所以, 在 $\tilde{J}\left(L^{k}(3)\right)$ 中, 有 $d^{e} S((n+1) r \sigma)-0$, 由定理 1 ,

$$
(n+1) S(r \sigma)=0 \text {. }
$$

再拉回 $\widetilde{K O}\left(L^{k}(3)\right)$ 中, 得到 $(n+1) r \sigma=0$, 于是 $(n+1) \equiv 0 \bmod 3^{\left[\frac{K}{2}\right]}$. 定理 2 得证.

\section{参參献}

[1] Kobayashi, T., Proc. Amer. Math.Soc., 97(1986), 714-716.

[2] Adams, J. F., Topology, 2(1963), 181-195.

[3] Kambe, T., J. Math.Soc. Japan, 18(1966), 135-146.

[4] Dai, Z. D., Lam, T. Y. et al., Extrait de L' Enseignement Math'ematique, 1981, 277-283.

[5] Adams, J. F., Topology, 3(1965), 137-171.

[6] Quillen, D., Topology, 10(1971), 67-80. 